\section{ECOFEMINISMO: UNA NUEVA MANERA DE MIRAR LA NATURALEZA}

\author{
María Antonia Bel Bravo \\ Universidad de Jaén \\ mabel@ujaen.es
}

Cómo citar este artículo/Citation: Bel Bravo, M.A. (2016). "Ecofeminismo: una nueva manera de mirar la naturaleza". Arbor, 192 (778): a304. doi: http://dx.doi.org/10.3989/ arbor.2016.778n2007

Recibido: 08 septiembre 2015. Aceptado: 14 septiembre 2015.

RESUMEN: El intento de erradicar la violencia en todas sus vertientes, que define a nuestro mundo, ha tenido su reflejo en el movimiento ecofeminista que, con diferentes perspectivas, ha tratado de conectar ambas cuestiones, feminismo y ecología por las muchas implicaciones que conllevan entre sí. En este artículo se analizan algunas de ellas desde una perspectiva interdisciplinar y se apuesta claramente por un ecofeminismo personalista y no constructivista.

PALABRAS CLAVE: Ecología; Feminismo; Naturaleza; Cultura; Educación.

\section{ECOFEMINISM: A NEW WAY TO LOOK AT NATURE}

Copyright: (C) 2016 CSIC. Este es un artículo de acceso abierto distribuido bajo los términos de la licencia Creative Commons Attribution (CC BY) España 3.0.
ABSTRACT: The attempt to eradicate violence in all its forms, which defines our world, has been reflected in the ecofeminist movement. From different perspectives it has tried to link the two areas, feminism and ecology, and study the many implications involved in this relationship. In this article, some of them are discussed from an interdisciplinary perspective and support is given to a clearly personalist and non constructivist ecofeminism.

KEYWORDS: Ecology; Feminism; Nature; Culture; Education. 
Hace unos años, en el seno del Seminario Interdisciplinar de Estudios sobre la Mujer de la Universidad de Jaén ${ }^{1}$, que yo dirigía en aquel momento, organizamos un curso para estudiantes universitarios que se titulaba Ecofeminismo: un reencuentro con la naturaleza y que iba en la línea de los estudios que estaba realizando sobre Historia de las Mujeres.

El título llamó tanto la atención que, a pesar de haber puesto un límite de 100 inscripciones, se nos matricularon 420 alumnos. Lo cual demostraba claramente por dónde iban las inquietudes de la gente joven. Sin embargo, en los debates que suscitaron las distintas intervenciones de los ponentes, se advirtió que aunque el tema interesaba mucho estaba un poco desenfocado.

Me explico: la idea que perseguíamos al organizar aquel curso era estudiar ese interés creciente que los temas ecológicos tenían y tienen en el mundo actual, y destacar a la vez la privilegiada relación que comparten con la mujer, por el hecho del superavit de experiencia que ella tiene en el cuidado y la conservación de la naturaleza en su totalidad: naturaleza humana y no humana. Cuidado y conservación que son valores en alza en este momento. Pero también se trataba de poner de manifiesto la incoherencia que supone defender a ultranza las cuestiones ecológicas, flora, fauna, clima, etc., y a la vez ignorar, o despreciar en muchos casos, al protagonista del medioambiente: el ser humano. Hambre, leyes abortistas y, sobre todo, mentalidad antinatalista así lo confirmaban.

Tema preocupante, porque se estaba generando entre los jóvenes una especie de esquizofrenia: defensa de "una" naturaleza sí, pero de otra no, y a cualquier educador le importa que sus alumnos tengan una personalidad coherente, es decir, madura. Significaba entre otras cosas que la educación adolecía de algún que otro fallo, por ejemplo la super especialización. Era urgente, por tanto, un diálogo entre las ciencias, porque cada una suele encerrarse en los límites de su propio lenguaje, y la especialización tiende a convertirse en aislamiento y en absolutización del propio saber. Esto es algo que, a mi juicio, impide afrontar adecuadamente los problemas de la vida en general, y en concreto los que aquí nos ocupan: los ecológicos.

En este sentido, al menos desde la Antropología fiIosófica, la Teología, la Historia y la Economía el tema requiere una perspectiva multidisciplinar.

\section{UNA MIRADA INTERDISCIPLINAR: ALGUNOS CONCEPTOS BÁSICOS}

\subsection{Antropología filosófica}

Siempre me ha llamado la atención que se denomine ingenuidad epistemológica al esencialismo que presuntamente profesan los seguidores de un primer ecofeminismo, entre los que me encuentro, y que algunos sustituyen por el constructivismo (Puleo, 2011). Pero vayamos por partes. Ingenuo es alguien que no sabe lo que es la "realidad", que no está en la "realidad" de las cosas, que, hablando coloquialmente, piensa que "to er mundo e gueno". Habría que plantearse en primer lugar qué entienden por "realidad" (de ahí las comillas) esos presuntos "realistas" ¿Solo lo que se ve, se oye, se palpa? Sería preciso entonces recordarles al escritor y aviador francés Antoine de Saint-Exupery cuando escribió, en El Principito, la famosa frase "lo esencial es invisible a los ojos", con cuyas palabras hace referencia a aquello que verdaderamente constituye a una persona: no su aspecto físico o su apariencia, sino su ser más íntimo: sentimientos, pensamientos, etc. En definitiva, cuestiones metafísicas.

El término "esencia" proviene del latín essentia, que a su vez deriva de un concepto griego. Se trata de una noción que hace referencia a lo característico y más importante de una cosa, que en muchos casos no se ve, pero que es tan real como lo que se puede ver, oler o tocar. Así pues, de acuerdo con esto, realista sería el que sabe que la esencia es aquello invariable y permanente que constituye la naturaleza de todo, lo cual no impide reconocer las muchas peculiaridades no esenciales que caracterizan a los seres vivos.

El cambio o la sustitución, digamos de este supuesto esencialismo, a un mal llamado e inicial constructivismo se produciría con la Modernidad ilustrada, siglos XVIII y XIX. Para el pensamiento ilustrado, fielmente expresado en la Enciclopedia, los conocimientos religiosos y metafísicos no son más que explicaciones ingenuas (esencialistas) que elabora el hombre no científico. Pero el progreso de la ciencia acabaría por iluminar todos los sectores y aspectos oscuros de la realidad, y mostraría la esterilidad de tales pseudociencias.

Augusto Comte, padre del positivismo cientifista, supuso que la humanidad atraviesa en su historia tres etapas sucesivas: la religiosa, la metafísica y la científica o positiva. Según él, el hombre primitivo ignora todo, teme todo y cree que las fuerzas de la naturale- 
za son dioses y espíritus superiores. Con el tiempo, la razón va depurando esta explicación politeísta hasta llegar a un solo Dios, concebido como supremo principio metafísico. Pero la evolución constante de la razón acaba por descubrir que la metafísica es irreal e innecesaria: para explicar totalmente el universo basta con el conocimiento científico basado en la observación de los hechos y en la deducción matemática.

Así pues, desde que nace la ciencia moderna con sus descubrimientos maravillosos, con leyes de una exactitud asombrosa y con el fruto sabroso de una técnica que eleva enormemente la calidad de vida, nace también la tentación de conocer toda la realidad con exactitud matemática. Y como ello no es posible, porque los problemas vitales no se resuelven al modo matemático, el precio que se paga por esa exactitud va a ser el reduccionismo, que ahoga la compleja exuberancia de lo vivo y de lo humano.

En gran medida, el problema de las/los que rechazan el esencialismo es ver naturaleza y cultura como opuestas, como antinómicas y no como diferentes y complementarias, que es lo que son. Es una visión del mundo que ve contradicciones allí donde solo hay contrastes, síntoma claro de mediocridad intelectual porque considerar la naturaleza como un principio contrapuesto al ser humano lleva consigo asumir que se trata de un enemigo al que hay que derrotar para obtener sus recursos y así no nos puede extrañar el lamentable estado en que se encuentran amplias zonas del planeta Tierra. Lo estudiaremos detenidamente más adelante.

Sabemos que la consideración de realidades como contrapuestas y enfrentadas: materia-espíritu, malbien, mundo-cielo... ha sido una postura recurrente a lo largo de la Historia. Gnósticos, maniqueos, cátaros, bogómilos y otros movimientos filosóficos han recogido este planteamiento dualista del mundo. En el plano de la conservación ambiental, considerar la naturaleza como un principio contrapuesto al ser humano lleva consigo asumir que se trata de un enemigo al que hay que derrotar para obtener sus recursos. Para Derrick (1987), un ensayista interesado en las conexiones entre teología y medio ambiente, buena parte del problema ecológico se relaciona con una concepción maniquea de la realidad material, que considera necesario transformar el estado natural para darle valor, ya que solo lo originado por el espíritu humano sería bueno. En otras palabras, lo natural sería un estado primitivo que habría que elevar al estadio de "civilizado" por la intervención humana, que fundamentaría su desarrollo en una permanente conquista de la naturaleza hostil.

\subsection{Teología}

En este sentido, también es necesario dialogar con la Teología ${ }^{2}$ ya que considerar al mundo como bueno en sí mismo porque ha salido de las manos de Dios, permite enfocar con una actitud positiva la cuestión ecológica, sabiendo que si el diseño original de Dios era bueno, nuestra intervención no necesariamente perfecciona la naturaleza (aunque tampoco tiene por qué ser siempre negativa). En suma, también los estados naturales, esos estadios no transformados tienen un valor en sí mismos para el ser humano, por ejemplo, para contemplar reflejos de la belleza divina, o para aprender más sobre el funcionamiento de nuestra realidad material. Ciertamente, el aprovechamiento de los recursos naturales implica un notable esfuerzo, sobre todo en aquellos períodos de la Historia, o aún hoy en ciertas regiones del planeta, con condiciones de supervivencia más precarias, pero esa contraposición hemos de enmarcarla en el desequilibrio moral causado por el pecado, y no en el designio original de Dios.

Así lo indicaba Juan Pablo II en la Centessimus Annus:

En la raíz de la insensata destrucción del ambiente natural hay un error antropológico, por desgracia muy difundido en nuestro tiempo. El hombre, que descubre su capacidad de transformar y, en cierto sentido, de crear el mundo con el propio trabajo, olvida que éste se desarrolla siempre sobre la base de la primera y originaria donación de las cosas por parte de Dios. Cree que puede disponer arbitrariamente de la tierra, sometiéndola sin reservas a su voluntad como si ella no tuviese una fisonomía propia y un destino anterior dados por Dios, y que el hombre puede desarrollar ciertamente, pero que no debe traicionar. En vez de desempeñar su papel de colaborador de Dios en la obra de la creación, el hombre suplanta a Dios y con ello provoca la rebelión de la naturaleza, más bien tiranizada que gobernada por él” (Juan Pablo II, 1991, n. 37).

Pero veamos algo de Historia.

\subsection{Historia}

A lo largo de la Historia ha habido muchas posturas que dan respuesta a por qué conservar la naturaleza, si bien han sido mayoritarios los planteamientos en los que la naturaleza se ha considerado una fuente de riqueza, y los recursos naturales simplemente como medios para satisfacer las demandas materiales del ser humano. En nuestra órbita cultural resulta relativamente reciente la preocupación por temas ambientales, tal y como los entendemos hoy. Ciertamente, 
desde la época clásica podemos rastrear estudios que podríamos denominar "naturalistas", pero realmente solo a partir de mediados del siglo XIX comienza el movimiento conservacionista propiamente dicho. LoS pioneros de esa corriente ideológica son tres pensadores norteamericanos, Emerson, Thoreau y Marsh, que plantean una crítica al desarrollo economicista que se produce en su país en esas décadas, basado en una transformación abusiva de la naturaleza. Ese caldo de cultivo se plasma en el nacimiento de los primeros grupos ecologistas (Sierra Club en 1892), y en la declaración de los primeros parques nacionales (Yellowstone, 1872). Buena parte de esta actividad se desarrolla en torno a las figuras de John Muir y Gillford Pinchot, uno más en la línea del ecologismo militante, y otro más orientado hacia la planificación ambiental.

En los años sesenta tuvo un impacto excepcional el libro de Rachel Carson, La primavera silenciosa, que denunciaba el impacto indirecto del $\mathrm{DDT}^{3}$ sobre la mortalidad de las aves, y supuso a la larga su prohibición en EE.UU. en 1972. Esto se consideró uno de los primeros éxitos del movimiento ecologista frente a la industria química. En las últimas décadas, esta preocupación ambiental ha adquirido tintes cada vez más fuertes, creándose incluso escuelas filosóficas que conceden a la naturaleza derechos equivalentes a los seres humanos. Junto a ello, la actividad de los grupos ecologistas ha sido cada vez más protagonista, lográndose introducir los aspectos medioambientales en prácticamente todas las áreas de la planificación territorial, al menos en los países desarrollados, donde el medio ambiente forma un pilar básico de eso que denominamos nebulosamente como "progreso".

Ahora bien, la situación ambiental del Planeta dista mucho de ser idónea, y todavía son múltiples los problemas creados por una actividad productiva no siempre asociada con la satisfacción de las necesidades básicas de los seres humanos. Las tremendas cifras de deforestación en los países en desarrollo, principalmente en las regiones tropicales, llevan consigo una pérdida de biodiversidad, degradación de suelos, deterioro de la calidad de las aguas y mayor impacto de las inundaciones. La contaminación del agua por vertidos industriales y el deterioro de acuíferos por sobre-explotación agrícola es un fenómeno bastante generalizado internacionalmente. Los incendios forestales alteran los ciclos naturales de regeneración, contribuyen a la degradación de los suelos y al aumento de la contaminación del aire. Los procesos de desertificación causados por la actividad humana también son cada vez más preocupantes. Finalmen- te, el reto actual que plantea detener las emisiones de gases de efecto invernadero va a llevar consigo un ingente esfuerzo por preservar las masas forestales y reducir la dependencia de los combustibles fósiles. El equilibrio entre el uso responsable de los recursos naturales y mantener un legado biológico a las generaciones futuras resulta cada vez más complicado, pero a la vez se subraya la importancia de lograr ese equilibrio para conseguir la tan deseada sostenibilidad de nuestro modelo de desarrollo económico. Parece pertinente, en este sentido, preguntarnos: ¿A lo largo de la Historia nadie se ha planteado cuáles son las implicaciones éticas de esa conservación de la naturaleza?, ¿qué principios filosóficos la fundamentan?, ¿cuáles son las actitudes propias de un ser humano responsable ante esa disyuntiva?

La cumbre de Copenhague 4 , que se celebró hace unos años, puso de manifiesto de nuevo estos problemas. So capa de una preocupación, completamente justificada por otra parte, por el hecho de que el medio ambiente se encuentre en un estado lamentable, al final ¿quién tenía, según los ilustres delegados, la culpa del desastre ecológico?, pues el exceso de población.

\subsection{Población/producción}

Pero no se trataba de los que ya estamos aquí, que somos los que nos lo estamos cargando, sino los que están por venir, los no nacidos. Es una manera como otra cualquiera de echar balones fuera: muerto el perro se acabó la rabia: cuantos menos comensales en el almuerzo de la vida, a más cabemos. Resucitado, una vez más, el falso dilema enunciado por Malthus en el siglo XVIII entre población y alimentos, entre población y producción, cuando a estas alturas es conocido por todos que el problema es esencialmente de distribución.

Es un tema antiguo, y descartado como falso por los más ilustres economistas, pero que resucita con periódica monotonía. Un reciente premio Nobel, Julian Simon ${ }^{5}$, ha dejado bien claro en sus trabajos que la auténtica riqueza de un país es su población ${ }^{6}$. Pero hagamos un poco de historia sobre este asunto.

Es Malthus quien por primera vez plantea el dilema "población-bienestar" en su obra Ensayo sobre el principio de la población que aparece por primera vez en 1798. En su libro apenas si tiene interés el aparente conflicto que plantea entre población y producción de bienes de consumo suponiendo sin mayores pruebas que en un proceso industrializador, la primera aumenta en proporción geométrica y la segunda en propor- 
ción aritmética y que, por consiguiente, para evitar los efectos inmorales del pauperismo no hay otro camino que la disminución de la natalidad en los medios obreros, acomodándola a la productividad económica.

Pero lo que importa es que Malthus quería que su argumento fuera un argumento contra la reforma social de su tiempo, nunca pensó en utilizarlo de ninguna otra manera. Sin embargo, el neomaltusianismo imperante traslada la cuestión al medioambiente ¿quién es el depredador?: el hombre, pues no permitamos que nazca siquiera.

Por otra parte, y en eso hay una similitud total con lo que sucede actualmente, al controlador de la natalidad de la época de Malthus no le interesaba controlar a los ricos; lo que deseaba era controlar a los pobres y así lo reconocía en la práctica. Siempre insistía en que un obrero no tiene derecho a tener tantos hijos, o que una barriada pobre es peligrosa porque produce tantos y tantos niños. La pregunta que le aterraba era ¿por qué el obrero no tiene un salario mejor?, ¿por qué la familia del barrio pobre no tiene una casa mejor? Su manera de evitarlas no era sugerir una casa más grande sino una familia más pequeña ${ }^{7}$.

No advirtieron, como señaló agudamente Chesterton, la contradicción implícita en esta cuestión: limitar el tamaño de las familias es una razón para disminuir los salarios y no una razón para aumentarlos. Si se puede hacer el salario más grande, no hay necesidad de hacer la familia más pequeña. Si se puede hacer la familia más pequeña, no hay necesidad de hacer el salario más grande. Origen, pues, del control de la natalidad, capitalista y reaccionario. Una violencia más sobre las clases débiles.

\section{FEMINISMO Y ECOLOGÍA}

Llegados a este punto podemos preguntarnos qué papel ha jugado y juega la mujer con respecto a los temas medioambientales, ecologistas, referentes a la naturaleza en general. La clave de la actuación histórica de las mujeres con respecto al dualismo naturaleza/cultura descansa en el hecho de que las actividades tradicionales de las mujeres: maternidad, cocinar, cultivar, curar, etc. son tanto sociales, es decir culturales, como naturales. La tarea de un feminismo ecológico es la configuración de una teoría y praxis antidualista. Los feminismos actuales no pueden tratar adecuadamente este problema desde el marco de sus teorías y políticas concretas, de aquí la necesidad del ecofeminismo, marco donde se use la mente y la historia para razonar desde el "ser" al "deber ser", para reconciliar a la humanidad con la naturaleza dentro y fuera. Este es el punto de partida de lo que se puede llamar ecofeminismo personalista:

Cada una de las principales teorías feministas contemporáneas -liberal, radical, socialista, cultural, etc.ha hecho suyo el problema de la relación entre las mujeres y la naturaleza, pero cada una de forma propia ha capitulado al pensamiento dualista" (King, 1998).

Desde una perspectiva ecofeminista todas son erróneas porque han escogido bien a la cultura bien a la naturaleza. Veamos por qué.

Como ya se ha apuntado más arriba, la idea de que la naturaleza es algo distinto a la cultura no es la única concepción posible de la naturaleza. Si algo pusieron de manifiesto Platón y Aristóteles, cuando calificaron al hombre de animal político, es que

la oposición abstracta entre la naturaleza del hombre individual, asocial, y la convención social de la realidad no era satisfactoria. Si se puede mostrar que el deseo del hombre de sobrevivir y de vivir bien se puede cumplir sólo en una forma social de vida, entonces cabe afirmar que la sociabilidad pertenece a la naturaleza del hombre (Bel Bravo, 1998, p. 24).

En los planteamientos clásicos, el concepto de naturaleza tiene un sentido dinámico, lo natural es lo que tiene en sí mismo un principio de movimiento y de reposo, lo que regula desde sí unas operaciones que pueden por ello calificarse en verdad como propias. La realidad es considerada por tanto dinámicamente: las cosas tienden naturalmente a algo que es su perfección. En su concepción clásica, la naturaleza tiene un sentido teleológico. Como los procesos naturales se orientan de suyo a un fin, la naturaleza presenta una teleología intrínseca (de suyo la bellota tiende a convertirse en encina, y la encina es el fin natural de la bellota, su perfección). Para el pensamiento clásico está dentro del ser lo que debe ser mientras que para el ilustrado solo está lo que es. Al considerar la realidad de un modo dinámico y no estático, en cuanto que la naturaleza es vida teleológicamente, en la medida en que se admite la existencia de procesos naturales dirigidos intrínsecamente a un objetivo, la clase o el tipo de cosa que algo es pasa a definirse desde su fin. "La naturaleza, escribe Aristóteles, es un fin y una causa final" para especificar que no todo lo que es término merece el nombre de "fin" sino solo el óptimo. La naturaleza de algo no queda determinada tanto desde su situación inicial cuanto desde su perfección final: las cosas son lo que serán cuando alcancen su plenitud; la realidad que algo es se define por la perfección que es capaz de alcanzar. 
El planteamiento de la Modernidad ilustrada es diferente. En primer lugar, la visión dinámica de la realidad ha sido sustituida por un planteamiento mecanicista en el que ya no caben fines intrínsecos. Donde el pensamiento clásico ve seres que tienden intrínsecamente a su perfección ya que para él entra en el ser también lo que "debe ser", el moderno advierte cuerpos físicos que se desplazan en el espacio movidos por fuerzas extrínsecas. En segundo lugar, y en consecuencia, la naturaleza de algo no queda fijada por un presunto estado de plenitud sino por su modo fáctico de ser. Las cosas son como son, y este modo de ser de las cosas, la forma en que de hecho funcionan y se organizan, constituye ahora su naturaleza. El pensamiento moderno que, desde Maquiavelo, interpreta la naturaleza como el hecho de ser como se es, la desimboliza: las cosas son exclusivamente lo que son; no significan nada. Como donde había tendencias ahora hay hechos, las cosas se agotan en ser lo que son.

Ahora bien, para definir qué sea lo natural resulta preciso averiguar cuál sea esa perfección y esa plenitud a la que se tiende. Con otras palabras: la concepción clásica de la naturaleza deja mucho espacio a la hermenéutica (es decir, a la interpretación). La distinción entre las operaciones propias de un ser y lo que meramente le sucede implica todo un contexto de normalidad. Necesitamos un contexto de lo que es normal, de lo que habitualmente sucede o de lo que debería ocurrir para considerar que el crecimiento de un árbol es natural mientras que su destrucción por un rayo es algo que accidentalmente le pasa.

La interpretación moderna de la naturaleza la presenta como un "sustrato" compuesto de hechos: las cosas ya no se definen por lo que presuntamente significan sino por ser como son. Y como de "hecho" son como son, la hermenéutica ${ }^{8}$ puede sustituirse por la ciencia positiva. La expresión "naturaleza humana" no significa, por tanto, lo mismo antes y después de la llustración. Porque, mientras en el enfoque clásico la naturaleza humana es sinónimo de la plenitud humana, en el planteamiento ilustrado se fija desde un funcionamiento presuntamente común a todos los hombres de todas las culturas. Si para Aristóteles lo natural es lo mejor de lo que el hombre es capaz, para los ilustrados es la manera de funcionar constante al género humano.

En consecuencia, mientras en la perspectiva clásica la noción de naturaleza humana incluye la de cultura -porque la conducta específicamente humana tiene como requisito un proceso de aprendizaje sociocultural y el hombre no puede realizar las actividades que le corresponden de suyo si no es en un medio cultu- ral-, la llustración tiende a oponerlas. Aristóteles llega a pensar en la cultura como una "segunda naturaleza" que se explica, o puede explicarse, con las mismas categorías que la naturaleza con la que parece estar en perfecta continuidad. Solo en la razón se manifiesta la naturaleza como naturaleza. Es la llustración, o la Modernidad como queramos Ilamarla, por tanto, la que "decide" un corte abrupto donde el planteamiento clásico ve una continuidad.

Jacinto Choza ha mantenido en diferentes trabajos (1992) que "la cultura es la verdad de la naturaleza", porque solo mediante la cultura, a través del trabajo y de la actividad humana, la naturaleza llega a ser todo lo que puede ser, alcanza su última realidad, la perfección que la define. La naturaleza humana solo se desvela, solo muestra su verdad en la cultura. Por su parte, Spaemann ${ }^{9}$ no deja de advertir en el mismo sentido que "lo racional es también, en primer lugar, llegar a descubrir la verdad de lo natural, y esta revelación radica en la teleología de la naturaleza". Lo que el hombre es solo queda patente tras el cuidado y el cultivo de sí, es decir, tras un proceso de socialización, y no mediante un presunto primitivismo espontáneo.

Como la naturaleza no está "antes de" la cultura, sino más bien se desvela en los desarrollos culturales que son los que posibilitan al hombre actuar como tal, no cabe una descripción de la naturaleza humana que no asuma ya categorías culturales y éticas. La concepción teleológica de la naturaleza excluye su comprensión cuasimecánica. Lo natural no se desvela "fuera" de los desarrollos culturales que posibilitan la conducta específicamente humana, sino justamente "en" ellos. Lo natural no es lo primitivo: es lo mejor. La relación entre naturaleza y cultura es, por tanto, hermenéutica.

"Humanizar es siempre dar sentido". El hombre humaniza el mundo externo en el que vive y su mundo interno: deseos, sentimientos, tendencias, aspiraciones, etc. cuando los interpreta, cuando les adjudica un significado de modo que pueda orientarse en ellos y saber dónde está, tanto respecto de la naturaleza externa como de su universo interno.

\section{ECOFEMINISMO PERSONALISTA}

A la mujer, circunscrita durante siglos a la casa y al ámbito de lo privado, se le adjudicaron unas cualidades (intuición, amor por lo concreto, cuidado de los detalles, espíritu de servicio para atender a las personas singularmente, etc.) que han consagrado "el eterno femenino" y que no dejan de ser un tópico. No obstante es posible que el tópico obedezca antes 
de haberse enquistado y falseado, a una realidad más original y profunda, como puso de manifiesta la profesora Carmen Segura en el congreso El espacio social femenino (Pamplona, 1995).

En efecto, no parece descabellado suponer que la peculiar relación que la mujer guarda con la vida haya generado en ella unas disposiciones particulares. Al reflexionar sobre su forma de vivir y sobre las funciones que ha desempeñado durante tantos siglos se entiende que haya desarrollado especialmente determinados hábitos intelectuales y capacidades: aquellos que tienen que ver directamente con la práctica. Frecuentemente su conocimiento se ha movido dentro del ámbito de lo que llamamos experiencia (que Aristóteles denominaba techné), puesto que, además, se le negó el acceso a la formación intelectual y al conocimiento científico.

Esto explicaría, por ejemplo, ese curioso fenómeno de la "intuición femenina", ese "ver" sin necesidad de discurso, esa inteligencia, que denomino "poliédrica" (Bel, 1998), porque es capaz de tener en cuenta todos los planos de la vida humana: no solo los intelectivos sino también los afectivos, que en tantos momentos condicionan de forma mucho más intensa a la persona. Por contraste, el hombre habría desarrollado, también durante generaciones, hábitos intelectuales más abstractos, los propios de la ciencia, no relacionados directamente con el cuidado del mundo de la vida. No se trata de que los hombres atiendan al desarrollo de capacidades "femeninas" ni de que las mujeres realicen lo correspondiente con las "masculinas"; sino más bien de desbloquear una reduccionista concepción de lo específico y de lo no-específico de los hombres y de las mujeres. Este es un postulado fundamental del ecofeminismo.

Es verdad que hubo durante siglos una gran despreocupación ecológica debido a la creencia en el carácter ilimitado de los recursos naturales. La carencia de conciencia de lo cualitativo, por la sola atención a lo cuantitativo, impedía en definitiva descubrir la distinción entre recursos renovables y no renovables, dado que solo importaba su valor monetario. Esta ideología exige, a su vez, un modo de organización social, que toma la precisión como modelo. El lema del organizador moderno es que la sociedad funcione como un reloj. De ahí que como ha escrito Schumacher, "el ideal de la Modernización industrial sería eliminar lo vivo, incluyendo lo humano, y transferir el proceso productivo a las máquinas, ya que estas pueden trabajar con más precisión y se las puede programar íntegramente, lo que no cabe hacer con el hombre" (1990, p. 78).
Juan Pablo II fue el primer papa en dedicar una encíclica a la ecología. Benedicto XVI también apostó por detener las emisiones tóxicas y su postura bien podría definirse como ecologista. $Y$ no digamos nada de la Laudato Si' (2015) del Papa Francisco que acaba de aparecer. Los tres han dejado claro que la doctrina católica sobre el medio ambiente puede resumirse así: la naturaleza está al servicio del hombre, no al revés. El ser humano, hijo de Dios, está obligado a no infligir al medio ambiente daños innecesarios por ser un don recibido de Dios y, sobre todo, para preservarlo, a fin de que pueda ser aprovechado por las nuevas generaciones, es decir, por otros seres humanos. Pero el objetivo es la especie humana.

Por el contrario, actualmente se pretende crear un nuevo credo, una nueva religión sincrética, de tendencia eco-panteísta que contempla al hombre como una especie animal más, a la misma altura que los animales, la flora, los océanos o la capa de ozono y que, por tanto, no tiene primacía alguna sobre las especies animales. Es más, resulta el enemigo a batir, en cuanto es el principal depredador y el causante de los males ambientales. En nuestras manos está poner las cosas en su sitio.

\section{UNA APUESTA POR OTRA EDUCACIÓN}

Ahora bien, la dificultad de plasmar las exigencias prácticas de estas propuestas radica en la necesidad de superación del espíritu de las dos instituciones básicas de la Modernidad, el nacionalismo del Estado -abriendo fronteras, trazadas tantas veces de forma convencional y arbitraria- y el consumismo del mercado -recuperando la noción clásica de economía como "arte de distribuir recursos escasos" y desechando de una vez la concepción mágica que hace del propio mercado un distribuidor milagroso de los bienes, cuestión esta que nunca se ha dado en la Historia-.

En definitiva, hay que ir más allá del mercado (para el que todo es venal, comprable) y del Estado (para el que todo es intrafronterizo) (Ballesteros, 1995). Es el modelo actual de las ONG. Para este modelo, la libertad coincide con la captación de la interdependencia y requiere un enorme esfuerzo intelectual y volitivo, ya que lo espontáneo es el egoísmo individual o colectivo. La solidaridad interdependiente es la base del movimiento de las ONG, en las que se trata al otro como fin y no como un medio susceptible de ser utilizado, manipulado.

El único modo de salvar conjuntamente los derechos de las mujeres, los niños y la naturaleza en general, es a través del reconocimiento por parte de los 
varones de que es necesario defender la cultura de la vida, como proponía Hannah Arendt (1998); que la política en vez de girar en torno a la mortalidad como hasta ahora (guerras, complejos armamentísticos, etc.), gire en torno a la natalidad; y no solo en torno a ella sino en torno a la fuerza de la razón (en lugar de la razón de la fuerza) ${ }^{10}$, la confianza en el otro y la voluntad de diálogo.

Solo si se respeta la vida humana desde la concepción hasta la muerte es posible y creíble también la ética de la paz; solo entonces la no violencia puede expresarse en todas las direcciones; solo entonces respetamos verdaderamente la creación; y solo entonces se puede llegar a la verdadera justicia. "Se trata ahora de reivindicar la primacía de los valores que han sido considerados hasta ahora como femeninos" (Ballesteros, 1989), pero que son en definitiva valores humanos, como la no violencia (a todos los niveles, también el doméstico), al igual que la atención a los más indigentes y menesterosos, a los más pobres, ya que ambas cuestiones están íntimamente entrelazadas, y competen tanto a hombres como a mujeres.

En este sentido, toda la Historia apunta al hecho de que es el hombre y no la naturaleza quien proporciona los recursos primarios, que el factor clave de todo desarrollo económico proviene de la mente del hombre. En algún momento de nuestra vida nos damos cuenta de que existe una explosión de fuerza, de iniciativa, de creatividad que, en principio, no sabemos muy bien de donde viene, pero muy pronto nos damos cuenta de que es la educación, el más vital de los recursos. Pero ¿qué educación?

Si la civilización occidental está en un estado de permanente crisis, no es nada antojadizo sugerir que podría haber algo equivocado en su educación. Ninguna civilización ha dedicado más energía y recursos para la educación organizada, y aunque no creyéramos absolutamente en nada, sí creemos que la educación es, o debiera ser, la llave de todas las cosas. En realidad, la fe en la educación es tan fuerte que la consideramos como la destinataria residual de todos nuestros problemas (Schumacher, 1990, p. 114).

Estamos ante un gran desafío educativo.

La tarea de la educación sería, primero y antes que nada, la transmisión de criterios de valor, de qué hacer con nuestras vidas. Sin ninguna duda también hay necesidad de transmitir el "saber cómo", pero esto debe estar en un segundo plano porque primero se ha de tener una idea razonable de qué hay que hacer. La esencia de la educación, pues, es la transmisión de valores porque cuando la gente pide educación lo que normalmente quiere decir es que necesita algo más que entrenamiento, algo más que el mero conocimiento de los hechos, algo más que un mero progreso técnico.

Se trata de redefinir el progreso porque "un desarrollo tecnológico y económico que no deja un mundo mejor y una calidad de vida integral no puede considerarse progreso" (Laudato Si', 2015, p. 174) ¿Qué es entonces la educación? Es la transmisión de ideas que le permitan al hombre elegir entre una cosa u otra y, en este sentido, apostar por una ética ecológica.

Así pues, la educación solo puede llevar el nombre de tal si produce hombres completos. El hombre verdaderamente educado no es aquel que sabe un poco de cada cosa, para eso ya están las enciclopedias. $\mathrm{Ni}$ tampoco lo es aquel que únicamente sabe cómo hacerlas. Una educación que solo le proporcionara esto (y no es mi intención restarle valor) sería meramente instrumental, mientras que lo que necesita el ser humano es la comprensión del por qué las cosas son como son y qué es lo que tenemos que hacer con nuestras vidas. El hombre educado, completo como lo llama Schumacher, es el que está en contacto con el centro. Es decir, el que no dudará acerca del significado y fin de la vida.

En esta línea, resulta urgente para el momento actual que los varones aprendan y asuman lo que se consideraban hasta ahora "valores de mujeres", muy especialmente el cuidado de la naturaleza, el cuidado de la paz y, en definitiva, el cuidado de todo aquello que contribuye a humanizar las relaciones sociales, a hacerlas más cultas, de mejores modos. Al mismo tiempo se trata de rechazar lo que se consideraba que era propio de varones, pero que en realidad era infrahumano, como la guerra o la violencia en general.

A mi juicio, tanto los hombres como las mujeres han desarrollado de forma reduccionista sus capacidades y han llevado a cabo una parcial comprensión del mundo. Es decir, no han desplegado plena ni adecuadamente su ser personal. Algo que ha supuesto un empobrecimiento para ambos y de lo que sería muy lamentable llegar a enorgullecerse. Se trata por tanto, y como ya dije más arriba, de desbloquear una concepción reduccionista de lo específico y de lo no-específico de los hombres y de las mujeres, que, entre otras cosas, contribuirá a un mayor cuidado de la Naturaleza. 


\section{NOTAS}

1. Seminario fundado a raíz de la IV Conferencia Internacional de la ONU celebrada en Pekín en 1995, que dejó abiertos multitud de temas para ser profundizados en sucesivos eventos. Uno de esos temas es el que nos ocupa en este artículo: mujeres y ecología.

2. En el campo mismo de la teología, no ha faltado quien haya hecho planteamientos al menos con la intención de suavizar el impacto de los supuestos adelantos modernos con bases y argumentos teológicos. En este sentido encontramos a fines del siglo dieciocho, al teólogo luterano John Bruckner (17261804), preocupado por el daño que los colonizadores en América del Norte infligían al medio ambiente al talar los bosques y así poner en peligro el mismo balance ecológico que en ellos había.

3. Dicloro, Difenil Tricloretano, principa insecticida del momento. Si bien este plaguicida fue muy útil para controlar muchas plagas en muy diversas zonas del planeta, en los años 1960 comenzaron a aparecer algunos datos negativos adjudicados al uso del DDT, que desataron la actividad de los primeros ecologistas en su contra.

4. Copenhague tenía como pretensión llegar a un acuerdo internacional para hacer frente al calentamiento global a partir de 2012 y reemplazar así el Protocolo de Kioto por un nuevo tratado vinculante, propósito fallido.

5. Julian Simon en 1980 escribió un artículo en Science titulado "Resources, Population, Environment: an Oversupply of False Bad News" que empezaba así: "Falsas malas noticias acerca del crecimiento de la población, los recursos naturales y el medioambiente son ampliamente publicadas en contra de toda evidencia. Por ejemplo, la superficie mundial de tierra cultivable ha estado

\section{BIBLIOGRAFÍA}

Agra Romero, M. J. (comp.) (1998). Ecología y Feminismo. Granada: Comares.

Arendt, H. (1998). Los orígenes del totalitarismo. Madrid: Taurus.

Ballesteros, J. (1989). Postmodernidad, de cadencia o resistencia. Madrid: Tecnos.

Ballesteros, J. (1995). Ecologismo personalista. Madrid: Tecnos. creciendo efectivamente, la escasez de recursos naturales incluyendo alimentos y energía ha ido menguando, y los indicadores básicos de la calidad ambiental de los EE.UU. muestran signos positivos. Los datos agregados no muestran ningún efecto negativo a largo plazo del crecimiento de la población sobre el nivel de vida. Modelos que encarnan fuerzas omitidas en el pasado, especialmente la influencia del tamaño de la población sobre el incremento de productividad, sugieren un efecto positivo a largo plazo de la población adicional".

6. La premisa central de Simon es que las personas son el recurso definitivo, así tituló su obra cumbre, The Ultimate Resource [El Último Recurso], Ed. Dossat, publicada en 1981 y actualizada en 1996 como The Ultimate Resource 2. El meollo del libro es bien claro: las reservas de recursos naturales no son finitas pues son creadas por el recurso siempre renovable de la inteligencia humana. En efecto, la madera, el carbón, el petróleo y el uranio no son recursos en absoluto hasta que no se combinan apropiadamente con el ingenio humano. "Los seres humanos," escribió, "no son meras bocas adicionales que alimentar, sino mentes productivas e imaginativas que ayudan a crear soluciones a los problemas humanos, dejándonos así en una mejor situación a largo plazo." Simon a menudo se preguntaba por qué la mayoría de las estadísticas gubernamentales sociales y económicas tratan a las personas como si de pasivos se tratara y no de activos. "Cada vez que nace un becerrillo", observaba, "el PIB per cápita de una nación aumenta. Cada vez que un bebé nace, el PIB per cápita cae."

7. El crecimiento de la población no es un obstáculo para el desarrollo económico, tal y como defiende la teoría malthusiana, sino que aumenta los estándares

Bel Bravo, M. A. (1998). La mujer en la Historia. Madrid: Encuentro.

Bel Bravo, M. A. (Coord.) (1999). Ecofeminismo. Un reencuentro con la naturale$z a$. Jaén: Universidad de Jaén.

Bel Bravo, M. A. (2000). La familia en la Historia. Madrid: Encuentro.

Bel Bravo, M. A. (2004). De la llustración al siglo XXI: una propuesta de trabajo. de vida a largo plazo. Los problemas no surgen tanto por el exceso de población como por la falta de libertad política y económica.

8. Se trata de un término con el que nos preguntamos por el proceso de la significación, por el carácter mediador de la inteligibilidad; como expresión o manifestación externa de una palabra interna, como interpretación de un enunciado que no se entiende por sí mismo, como traducción de un lenguaje extraño al lenguaje familiar.

9. El tema de la verdad y la lucha contra el relativismo es una constante en los escritos de Spaemann. Este relativismo se esconde habitualmente detrás de la palabra 'tolerancia', y la verdadera tolerancia, en cambio, presupone que hay convicciones; que son algo valioso para el hombre. Hoy, en nombre de la tolerancia, se prohíben las convicciones. $\mathrm{Si}$ hoy alguien manifiesta una convicción firme, se le llama intolerante.

10. Teoría esta de la "razón de la fuerza" ampliamente difundida por Hobbes y aplicada durante toda la Edad Moderna y Contemporánea, con los resultados de violencia sobre la naturaleza que todos conocemos. Los contactos que Hobbes tuvo con científicos de su época, que fueron decisivos para la formación de sus ideas filosóficas, le llevaron a fundir su preocupación por los problemas políticos y sociales con su interés por la geometría y el pensamiento de los filósofos mecanicistas. Su pensamiento político pretende ser una aplicación de las leyes del mecanicismo a los campos de la moral y la política. Las leyes que rigen el comportamiento humano son, según Hobbes, las mismas que rigen el universo, y son de origen divino, cuestión esta que, como ya se ha señalado, está abocada al fracaso.

En Puleo, A. H., Segura, C. y Cavana, M. L. (coords.) (2004). Mujeres y Ecología: Historia, Pensamiento, Sociedad. Madrid: Asociación Cultural Al Mudayna.

Bourdieu, P. (1997). Capital cultural, escuela y espacio social. Madrid: Siglo XXI.

Carson, R. (2010). Primavera silenciosa. Barcelona: Crítica. 
Castilla, B. (1992). La complementariedad varón-mujer: nuevas hipótesis. Madrid: Rialp.

Chinchilla, N. y León, C. (2004). La ambición femenina. Madrid: Aguilar.

Choza, J. (1992). Filosofía del hombre. Una antropología de la intimidad. Madrid: Rialp.

Derrick, Ch. (1987). La creación delicada. Una contribución cultural contra la destrucción del ambiente. Madrid: Encuentro.

D’Entremont, A. (1994). La ONU y la ideología neomalthusiana. Nuestro Tiempo, 484, pp. 70-79.

Francisco (2015). Encíclica Laudato Si'. Disponible en vatican.va.

George, R. P. y Elshtain, J. (2005). The Meaning of Marriage: Family. State, Market and Moral. Princeton.
Hymowitz, K. (2006). Marriage and Caste in America. New York: Manhattan Institute.

Jacobs, J. (1961). The Death and Life of Great American Cities. New York: Random House.

Juan Pablo II (1991). Encíclica Centessimus Annus. Disponible en vatican.va.

King, Y. (1998). Curando las heridas: feminismo, ecología y el dualismo naturaleza/cultura. En: Agra Romero, M. J. (comp.). Ecología y feminismo. Granada: Comares.

Malthus (1798/1998). Ensayo sobre el principio de la creación. México: Fondo de Cultura Económica.

Puleo, A. H., Segura, C. y Cavana, M. L. (coords.) (2004). Mujeres y Ecología: Historia, Pensamiento, Sociedad. Madrid: Asociación Cultural Al Mudayna.
Puleo, A. (2011). Ecofeminismo para otro mundo posible. Madrid: Cátedra.

Schumacher, E. F. (1990). Lo pequeño es hermoso. Madrid: Herman Blume.

Shiva, V. (1989). Las mujeres en la naturaleza. En: Agra Romero, M. J. (comp.). Ecología y feminismo. Granada: Comares.

Segura, C. (1999). El espacio social femenino. Pamplona: Eunsa.

Spaemann, R. (2000). Personas. Acerca de la distinción entre "algo" y "alguien". Pamplona: Eunsa.

Vicente Arregui, J. y Rodríguez Luesma, C. (1995). Inventar la sexualidad: sexo, naturaleza y cultura. Madrid: Rialp. 